\title{
LONDON BUS TUNES: USING SOUND TO IMPROVE THE SAFE NAVIGATION OF LONDON'S BUS SYSTEM
}

\author{
Dr Sara Adhitya \\ University College London, \\ Department of Civil, Environmental and Geomatic Engineering \\ London, WC1E 6BT, UK \\ s.adhitya@ucl.ac.uk
}

\begin{abstract}
This work-in-progress introduces a proposal to incorporate sound in the passenger navigation system of the London Bus. First, we present the problems of accessibility concerning London's complex bus system. Then, we introduce our proposal of using sound and sonification in particular to aid in the navigation of London's bus system. We explain our sonification strategy and describe a recent preliminary trial of our sonification prototype, implemented as an installation during an accessibility event held by Transport for London at the ExCel centre in London on 19 March 2019. We discuss the feedback obtained from this trial and conclude with proposed future work in terms of both the development of our sonification strategy as well as its implementation in London's public transport system.
\end{abstract}

\section{INTRODUCTION}

Consisting of 20,000 bus stops and 800 bus routes, navigating London's bus system can be a complex task for most passengers. Due to the sheer number of connections, getting on the right bus and off at the right stop can be difficult whether you are: unfamiliar with the city in general; travelling a new route; half asleep after a hard day's work; a non-English speaker; suffering from navigational challenges due to memory loss such as dementia; struggling to see in an over-crowded bus; and not least with a visual impairment. While there are many mobile navigation systems on the market developed specifically for the visually-impaired, there are clear benefits for all passengers in improving the overall legibility of the bus infrastructure system.

On 19 March 2019, Transport for London (TfL) [1], the city's integrated transport authority, held its biggest and most accessible transport event entitled Access All Areas at the ExCel centre. This event aimed to showcase innovative ways in which London's public transportation could be made more accessible. Furthermore, TfL has recently prioritised the safety of riding London's buses, as outlined under the Vision Zero action plan of its Transport Strategy [2], with the aim to eliminate serious injuries or death on all London Buses by 2030 [3]. With almost $80 \%$ of casualties from accidents involving buses consisting of bus occupants or pedestrians [3], improving the accessibility and legibility of the system clearly has an important role to play in achieving this goal.

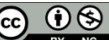

The full terms of the License are available at

http://creativecommons.org/licenses/by-nc/4.0/
Thus in this paper, we propose the use of sound to improve the safe navigation of London's bus system.

\section{BACKGROUND: Sound and urban transportation}

Sound is essential to navigation, particularly when vision is occupied or impaired, yet is an underutilised medium in the design of London's public transport system. While there is an announcement system in place on both tube trains and buses, this is limited to spoken announcements which can be difficult for non-English speaking passengers to understand as well as to hear in the context of noisy environments. Furthermore, psychoacoustic studies indicate that a difference in timbre is needed in order to distinguish between different auditory streams, which makes speech-based auditory cues problematic in crowded environments. Thus we propose the use of non-verbal auditory cues.

Non-verbal auditory cues have proven to be useful not only in helping navigation, but in contributing to the overall soundscape as well as creating a sonic identity for urban transportation systems. One notable example is the Yamanote train line in Tokyo, Japan, which has become famous for its musical tunes used to announce each stop. These tunes have also been recognised to have the added benefit of calming down pedestrian traffic flows and leading to a reduction in accidents [4].

London's bus network spans a much wider area than a tube network can, and thus can reach an even wider population. Yet there is currently no strategy concerning the consistency of its sound design. Thus we propose the development of a sound design strategy using non-speech auditory cues, not only to aid in the navigation of London's bus system, but develop its sonic identity while contributing positively to the overall urban soundscape.

\section{THE ISSUES}

In navigating any bus system, there are two key points of interchange that can be seen as problematic: a) boarding a bus; and b) alighting at a bus stop. The top three causes of casualties involving buses are due to standing, boarding and alighting [3]. In this section, we discuss the navigation and safety issues during boarding and alighting.

\section{a) Signalling and boarding a bus}

When waiting at a bus stop, it can be very difficult to identify which bus is arriving from a distance due to issues of legibility. Often the wrong bus is flagged down unnecessarily 
or at the last minute, leading to abrupt breaking and an increase in accidents. Many road accidents are caused by the bus veering and hitting pedestrians or cyclists. It is not uncommon that passengers board the wrong bus and realise only after it has begun moving.

\section{b) Signalling and alighting from a bus}

Once on the bus, it can be very difficult to know when to ring the bell and at which stop to alight. Passenger uncertainty often results in signalling the wrong stop, causing unnecessary breaking. Speaking to the driver whilst still on the move can also be distracting to the driver, and getting up earlier than necessary has been shown to increase the rate of falls. More than double the casualty value of injuries have been shown to occur while standing as opposed to being seated during a collision [3].

\section{PROPOSAL}

To mediate the navigation and safety problems encountered at these two points of interchange, in this section we propose the use of auditory cues in 2 main ways:

\subsection{Indicating which bus is arriving at a bus stop}

By announcing the arrival of a bus using sound from speakers within the bus stop, we can help the passenger know when to signal the bus. This can help in the case of inability to see the bus number while ensuring adequate time for the driver to stop. When the bus doors open, the same tune will be played from the bus itself, ensuring that the correct bus is boarded in the case of multiple arriving buses.

\subsection{Indicating which bus stop is approaching}

While there are currently spoken announcements which announce each stop, these can be difficult to distinguish and understand, particularly if English is not the first language or the background noise is high. By indicating the approaching bus stops using non-verbal auditory cues, we aim to improve the recognition of stops, and thus the timing of signalling and the preparation of alighting.

\section{METHODOLOGY}

Due to the large dataset of 20,000 bus stops and 800 bus routes, we chose to use the acoustic communication technique of sonification: the representation of data in sound [5]. In particular, we utilised the method of parametermapping, involving the mapping one of set of parameters to an audible set of parameters.

With the need to identify each bus and bus stop in the system, we utilised TfL's own identification system of 1 to 3 digit numbers for individual bus routes, and 5 digit numbers for individual bus stops. We then mapped each digit of each number to a specific tone. With 10 different digits to map, we utilised a 9-note blues scale: a chromatic variation of the major scale with a flattened third and seventh [6] which is shown in Figure 1 and can be heard at the following link:

https://www.dropbox.com/s/0d2kzha5cxlppp6/9\%20note $\% 20$ blues $\% 20$ scale.mp3? $\mathrm{dl}=0$

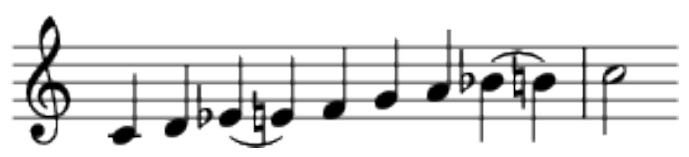

Figure 1: 9-note blues scale [7]

This gave us 10 different pitches which could be used to map each digit, and allowed us to generate a unique 1 to 5 digit auditory cue for each. At this preliminary stage, we utilised acoustic modes of sound production involving improvisation of a tune based on these notes and played on a flute.

For example, Bus 460 would be based on the notes E, G, C and sound like this:

https://www.dropbox.com/s/smnmhrms5x1yxlb/bus $\% 20460$ $\% 20-\% 20$ EGC.mp3?dl $=0$

whereas Bus stop 58903 would sound as follows: https://www.dropbox.com/s/ouxupq $7 \mathrm{kxnw} 49 \mathrm{tp} / \mathrm{Bus} \% 20$ stop \%2058903.mp3?dl=0

\section{INSTALLATION DESIGN}

Transport for London invited us to exhibit our proposal at their recent Access All Areas (AAA) exhibition [8], aimed at showcasing more accessible public transport to people of all abilities. Held at the ExCel Centre in March 2019, and with over 1500 attendees on the day, the AAA exhibition was an opportunity to introduce our idea to a large range of people of all capabilities, gauge interest and receive both spoken and written feedback.

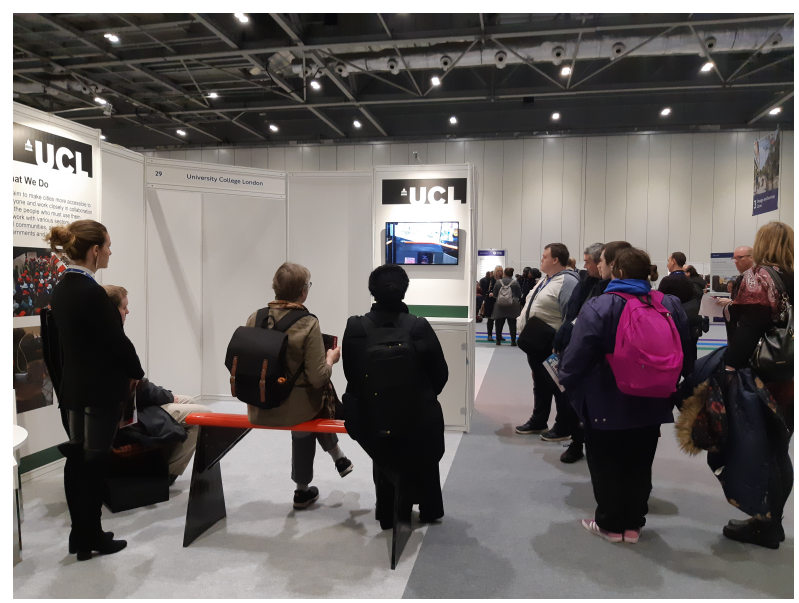

Figure 2: Sound installation at the AAA exhibition, ExCel Centre, London, 19 March 2019

We were provided with a $3 \mathrm{~m}$ by $6 \mathrm{~m}$ exhibition space in which to communicate our idea. In order to present our sonification proposal with as much context as possible, we created a sound installation in which the participants could imagine themselves in each scenario: a) seated at a bus stop while waiting for a bus; and b) seated on a bus while waiting to alight. The scenarios were displayed in video format on a digital screen provided by the organisers. 


\subsection{Scenario 1: view from the bus stop}

The first scenario showed two buses (Bus 325 and Bus 241) arriving at a bus stop in Stratford, east London. After introducing the sounds of each bus (film available here: https://www.dropbox.com/s/yxwvowqiunr2k93/Scenario $\% 20$ $1 \% 20-\% 20$ learn $\% 20$ bus $\% 20$ sounds.mp4?dl=0 )

, the participants were asked to identify a particular bus arriving by ear (audio file available here: https://www.dropbox.com/s/as1sy9jx02v93gw/Bus\%20241\% 20is\%20arriving.mp3?dl=0 ).

\subsection{Scenario 2: view from the bus seat}

The second scenario involved sitting on a bus seat and watching a journey filmed from the front of the bus with the sonified bus stops accompanying the announcement of bus stops. The video can be watched here: https://www.dropbox.com/s/860vr53o7gxag9g/Bus\%20241\% 20Stratford\%20-\%20Plaistow\%20Grove.mp4?dl=0

\section{FEEDBACK}

The exhibition was clearly not under controlled acoustic conditions, held within a large hall with a large number of other exhibitors in close proximity as well as sound permeating from the main auditorium. However, in spite of this noisy environment, we received a number of comments that the sound of our installation carried well above the various lectures and background chatter. Thus the location of the installation was useful in proving that the sound could be easily heard even in crowded environments, similar to the scenario of catching public transport.

We used the opportunity of having a large range of capabilities at hand to collect feedback from participants at the event. While providing feedback was optional, we obtained 34 survey forms from participants of a variety of capabilities ranging from bus drivers and operators, to the elderly and visually impaired. With respect to each scenario of identifying the bus route and bus stop, we asked participants how easy or hard it was to both recognize and remember the sounds on a scale of 1 to 5 .

\subsection{Scenario 1}

The majority of participants gave an intermediate response for both ease of remembering and recognizing the sounds. with many stating that simply needed the time to learn the tunes. This was to be expected, given that the installation only played each sound twice. Most said that it would help with catching the right bus with the main concern being when more than one bus arrives. Another was audibility if the ambient noise was too high. Several participants claimed specifically that they were struggling to see the bus numbers and that the sounds would help them.

\subsection{Scenario 2}

Remembering and recognizing the bus stop sounds proved to be more difficult. This was also expected due to the fact that there were more of them to select from and only the selected bus stop was played twice. Again, this was expected to improve by repetition and learning. Furthermore, it would not be expected to learn all the bus stop sounds, but rather the one required.

\subsection{Overall Feedback}

Finally, we asked participants what they thought of our proposed sonification based on the 9-note blues scale. We received mostly positive but generalized comments such as good, fantastic, nice, pleasant, calming, relaxing, distinct, enjoyable and innovative. Since the range of disabilities at the event were broad, there was some concern for hearingimpaired or 'tone-deaf' people. However, even though some did not think it would help their own navigation due to not being 'musical enough', they still commented on the music making their bus journey more pleasant.

We also collected feedback on what other sounds they would like to hear, and received suggestions such as bells or sirens, animal noises such as dogs barking and birds tweeting, the use of different instruments or lower pitches, and the use of more popular tunes or songs. A number of participants suggested location specific sounds, which would make more sense for more well-known places. There were a few which still preferred spoken announcements, but who specified that they should be clearer and more audible, suggesting the current inadequacies of the announcement system.

\section{FUTURE WORK}

As a result of the event, we were invited to present our sonification proposal at Transport for London's recent Bus Safety Innovation Challenge, which involved showcasing it to the various bus operators of the London bus network. We are now in the process of discussing the potential implementation of our work with several bus operators on the existing passenger announcement system. This would provide a relatively low-cost approach to testing the impact of the use of sonification in the bus system and we look forward to exploring this in the future.

\section{CONCLUSIONS}

While there is still much work to be done concerning the choice of sounds, including more controlled acoustic testing with various sectors of the population, the interest shown thus far in our proposed sonification of London's bus network is promising. We are currently in the process of exploring other sonification options, including other types of scales for parameter-mapping, as well as the use of different timbres and rhythms. Given the suggestion for clearer spoken announcements, we also intend to investigate the use of Spearcons [9]. We greatly look forward to the suggestions and feedback of the ICAD community.

\section{ACKNOWLEDGMENT}

We would like to thank Transport for London for funding our exhibition at the Access All Areas exhibition held at ExCel centre on 19 March 2019. We would also like to thank them for the invitation to present at the Bus Safety Innovation Challenge. 


\section{REFERENCES}

[1] Transport for London, www.tfl.gov.uk, Accessed 3 May 2019

[2] Transport for London, The Mayors Transport Strategy, https://tfl.gov.uk/corporate/about-tfl/the-mayorstransport-strategy, Accessed 3 May 2019

[3] Transport for London, Bus Safety Standard Executive Summary, 2018 http://content.tfl.gov.uk/bus-safetystandard-executive-summary.pdf, Accessed 3 May 2019

[4] A. Richarz, The Amazing Psychology of Japanese Train Stations, CityLab, 22 May 2018, Accessible at: https://www.citylab.com/transportation/2018/05/theamazing-psychology-of-japanese-train-stations/560822/

[5] W. Gaver, "How do we hear in the world? Explorations in ecological acoustics", Ecological Psychology 5, no.4, pp. 285-313, 1998

[6] S. Benward, Music: In Theory and Practice, vol. 1, p.39. Seventh Edition, 2003

[7] Wikipedia, Blues Scale, 19 November 2019, https://en.wikipedia.org/wiki/Blues scale\#Nonatonic, Accessed on 3 May 2019

[8] S. Brewster, "Using nonspeech sounds to provide navigation cues," ACM Trans. Comput.-Hum. Interact., vol. 5, no. 3, pp. 224-259, 1998 